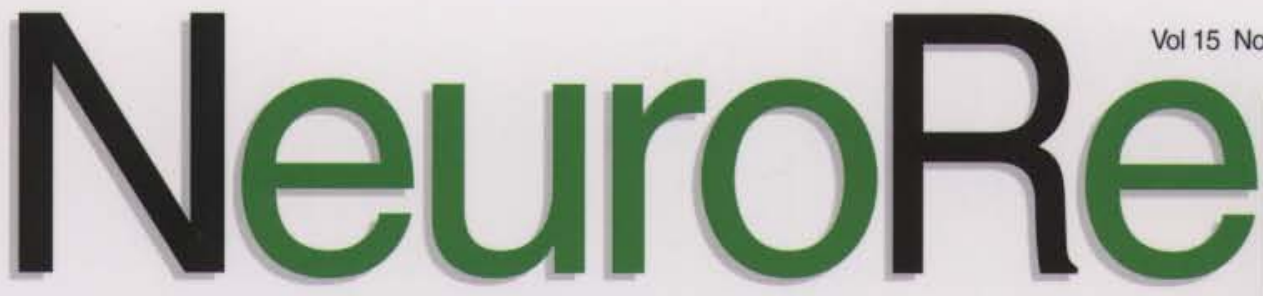

For Rapid Communication of Neuroscience Research

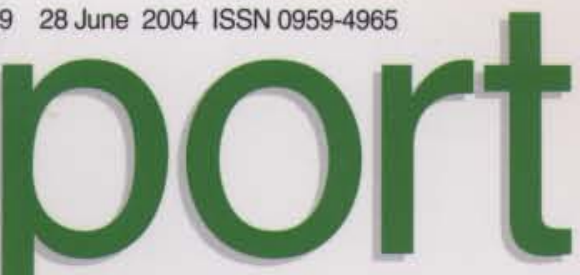

www.neuroreport.com

New: Online submission and review for NeuroReport at http://nr.edmgr.com

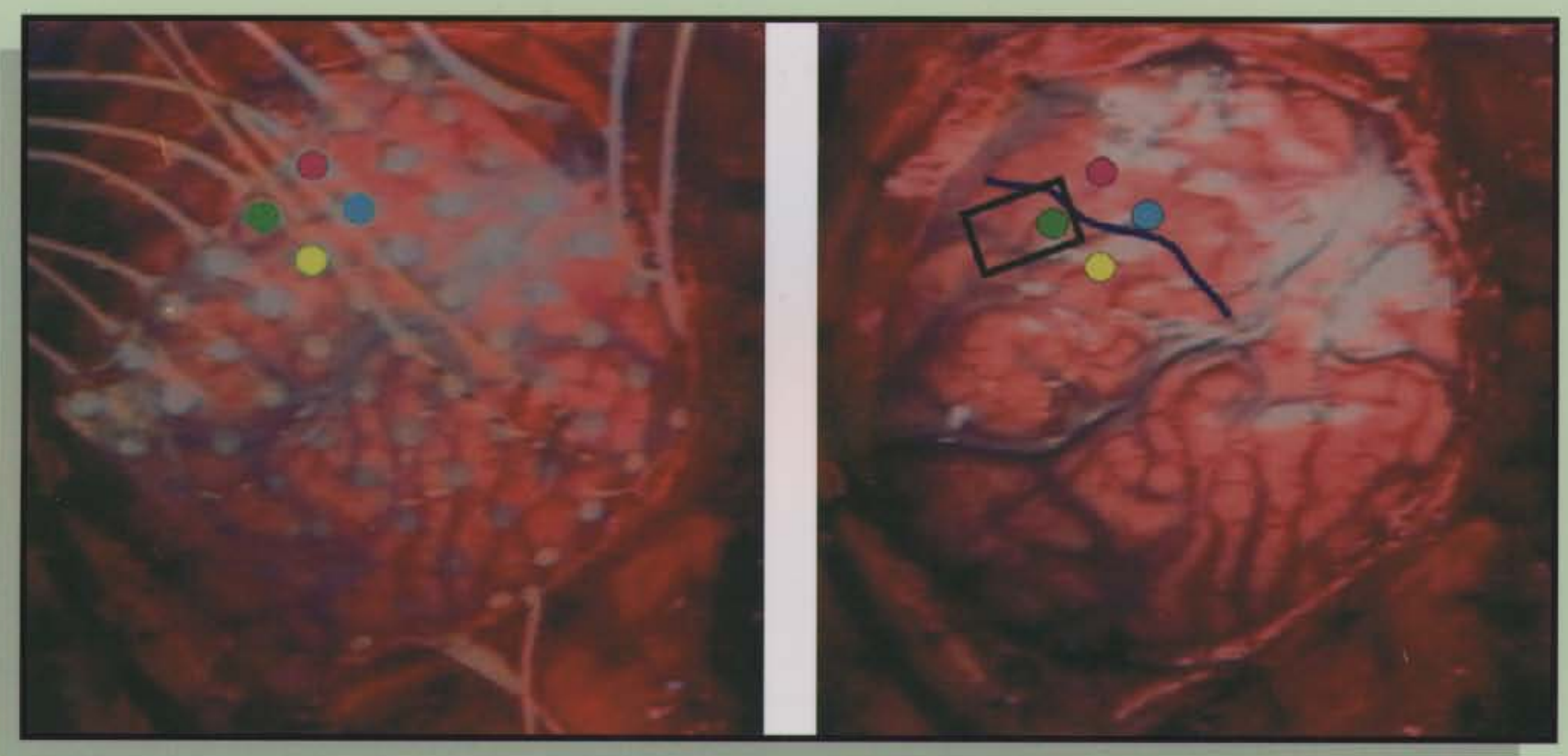

Human brain with surface electrodes reveals sites where stimulation elicited face sensation see inside back cover 


\title{
Intraoperative optical imaging of human face cortical topography: a case study
}

\author{
Theodore H. Schwartz, ${ }^{1,4}$ Li Min Chen, ${ }^{2}$ Robert M. Friedman, ${ }^{3}$ Dennis D. Spencer' and \\ Anna W. Roe ${ }^{2, C A}$
}

\begin{abstract}
Departments of 'Neurosurgery; ${ }^{2}$ Neurobiology; ${ }^{3}$ Anesthesiology, Yale University School of Medicine, New Haven, CT; ${ }^{4}$ Department of Neurological Surgery, Weill Cornell Medical College, New York Presbyterian Hospital, 525 East 68th St., Box \#99, New York, NY I002I, USA

${ }^{\mathrm{CA}, 4}$ Corresponding Author and Address: schwarh@med.cornell.edu
\end{abstract}

Received I2 March 2004; accepted 31 March 2004

DOI: 10.1097/0I.wnr.0000131006.59315.2f

\begin{abstract}
We used intrinsic signal optical imaging to obtain maps of human somatosensory cortex during electrocutaneous stimulation of the face during a neurosurgical procedure for epilepsy. We found that human face somatotopy is organized like the macaque or cebus monkey, with peri-orbital skin located medial to peri-buccal skin, and that cortical magnification in the human is comparable to that
\end{abstract}

in non-human primates. This study demonstrates that intrinsic signal imaging can be performed on humans during operative procedures with sufficient spatial resolution to reveal high-resolution topographic maps. NeuroReport 15:I527-1531 (C) 2004 Lippincott Williams \& Wilkins.

Key words: Cerebral cortex; Cortical maps; Intrinsic signals; Neurosurgery; Somatosensory

\section{INTRODUCTION}

Optical recording of intrinsic signals (ORIS) is an imaging method that detects tissue reflectance changes resulting from neural activity. These reflectance changes can be mapped under a range of illumination wavelengths (e.g. $570-800 \mathrm{~nm}$ ), different ranges of which accentuate different sources of the signal (e.g. changes in blood volume, oxygenation of hemoglobin, light scattering). Under laboratory conditions, ORIS provides high spatial resolution maps $(<100 \mu \mathrm{m})$ [1]. However, there are limitations inherent in the operating room related to mechanical and biological noise, which lead to poorer signal to noise ratio and poorer spatial resolution than in the laboratory. Despite this, several groups have successfully utilized ORIS in the operating room to map human cortex [2-6].

The purpose of this study was to obtain intrinsic signal optical maps from human sensory cortex in the neurosurgical arena and to do so with sufficient resolution to map the representation of upper and lower face. To minimize motion artifacts and vascular noise, we implemented some methodological changes in the use of ORIS in the operating room environment, which included mechanical stabilization of the brain, tandem camera lens arrangement, and the use of longer wavelength illuminant. We demonstrate that focal activations are obtainable with intraoperative optical imaging methods and that the upper face is represented medial to the representation of the lower face. We also show that cortical magnification factor $(\mathrm{CMF})$ in the human is comparable to other non-human primates.

\section{MATERIALS AND METHODS}

The experiment was performed under a protocol approved by Yale Human Investigational Committee. The subject was an adult male (age 39 years) who underwent extraoperative stimulation mapping of the right somatosensory cortex with a chronically implanted grid of surface electrodes (Ad-Tech, Racine, WI) as part of investigations for surgical treatment of medically intractable epilepsy. Bipolar stimulating electrodes $(50 \mathrm{~Hz}, 0.1 \mathrm{~s}, 4-8 \mathrm{~mA})$ were used to identify the face representation in motor and sensory cortex.

Electrical grid stimulation revealed the facial topography in this region (Fig. 1a). Four sites are highlighted in Fig. 1b,c. At two sites (yellow and green dots) the subject reported sensations located on the left lower lip. In two rostral sites (red and blue dots) twitching of the lip was elicited. These observations indicated the border between facial somatosensory and motor representations. We positioned the imaging field of view slightly medial to the somatosensory representation of the lower face/mouth area to include the presumed representation of the upper face (black box in Fig. 1b, shown enlarged in Fig. 2d).

During the surgical procedure, a $4 \times 4 \mathrm{~cm}$ glass footplate (Fig. 1d), held by a retractor arm attached to the Mayfield head-holder, was placed on the cortex. The optical imaging camera (Imager 2000 system, Optical Imaging Inc., Germantown, NY, USA) was held by a custom-built sterilizable camera-holder secured to both the operating table and the Mayfield head-holder. In this way there was minimal relative movement between the cortical surface and the camera lens. A tandem lens macroscope, designed to maximize the field of view $(1-2 \mathrm{~cm})$ and minimize the 
(a)

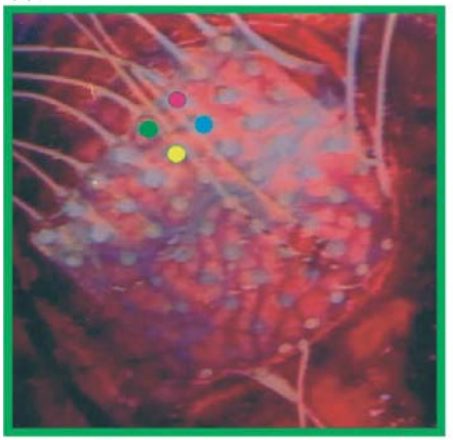

(c)

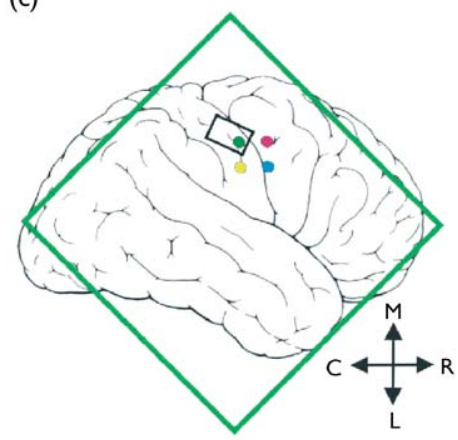

(b)

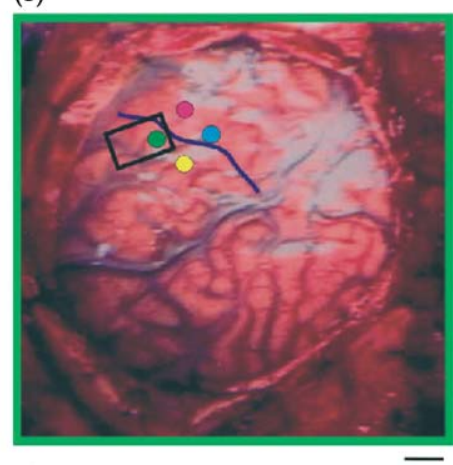

(d)

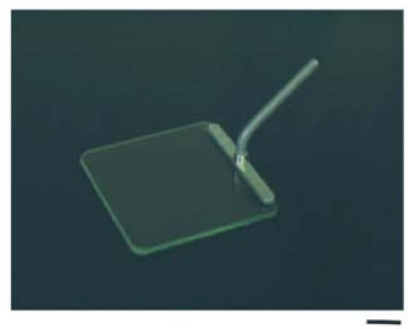

Fig. I. Extraoperative grid stimulation identifies face somatosensory cortex. (a) Location of the 64-contact grid (I cm spacing) on brain. Stimulation between the green and yellow contacts $(3 \mathrm{~mA})$ elicited sensations at the edge of the left lower lip. Stimulation between the blue and red electrodes $(3 \mathrm{~mA})$ elicited twitching of the left upper lip. (b) Location of the optical window relative to the electrode contacts. The central sulcus is highlighted in dark blue. (c) Orientation of the optical window and electrode contacts. Orientation of green frame in (a) and (b) shown. (d) Glass footplate is placed gently on the cortex for stabilization. Bars =I cm. R-rostral; C-caudal; M-medial; L-lateral.

(a)
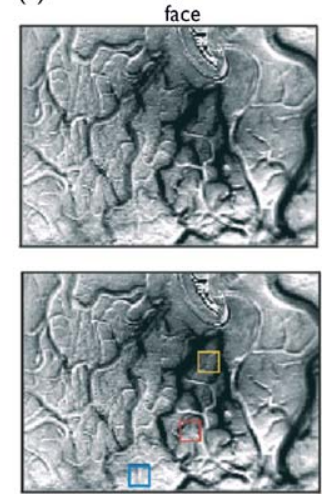

(e)

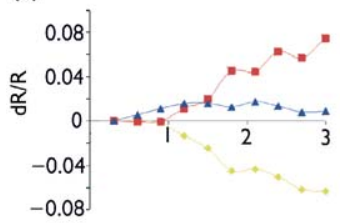

(b)
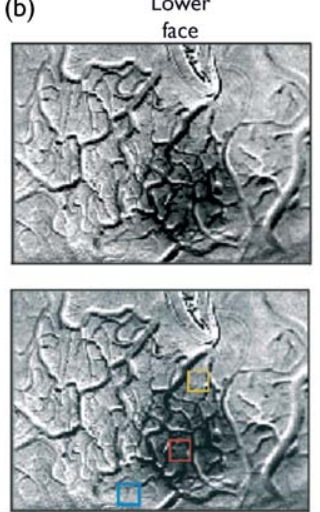

(f)

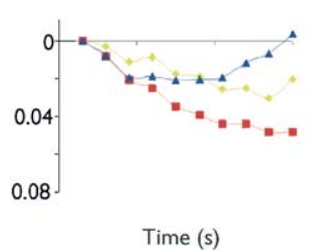

(c)
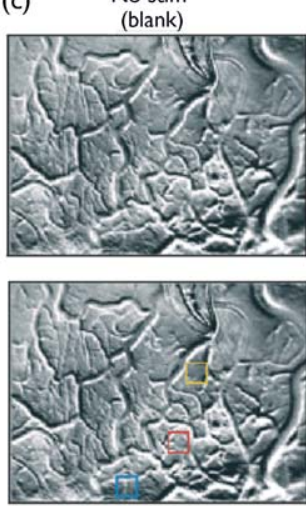

(g)

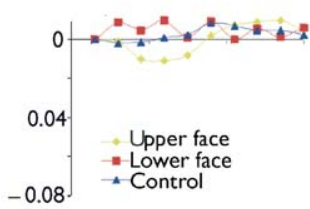

(d)
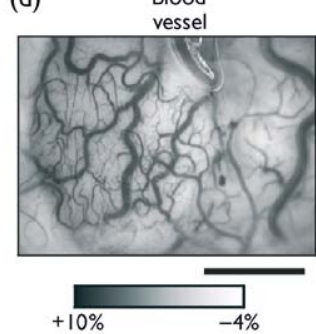

Upper face location

Lower face location

Control location

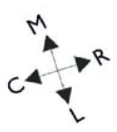

Fig. 2. Optical imaging of human face region. (a-c) Top, raw blank-subtracted image. Sum of 5 trials. Bottom, same image with sampled signal locations shown. (a) Activation to upper face stimulation. (b) Activation to lower face stimulation. (c) Blank (no stimulation) condition. (d) Blood vessel map of imaged field of view. (e) Stimulation of upper face produced three different reflectance signal timecourses at three different locations: a large reflectance change over the upper face location (yellow), smaller reflectance change over the lower face location (red), and little change over the control location (blue). (f) Timecourse of signals obtained during stimulation of lower face. Same sites as in (e). Activation of lower face produces large reflectance change over lower face location (red), smaller reflectance change over upper face location (yellow), and little change over control location (blue). (g) Timecourse of signals obtained during blank condition. Same sites as in (e). Bar $=5 \mathrm{~mm}$.

1528

Vol 15 No 928 June 2004

Copyright ( $)$ Lippincott Williams \& Wilkins. Unauthorized reproduction of this article is prohibited. 
(a)

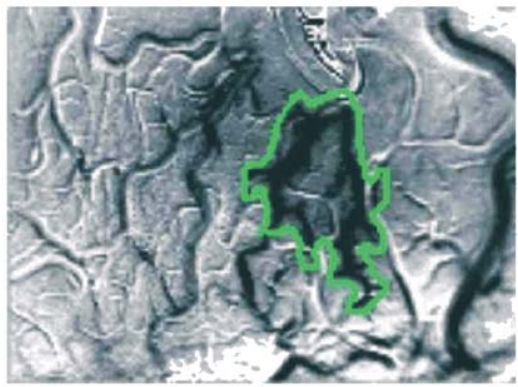

(b)

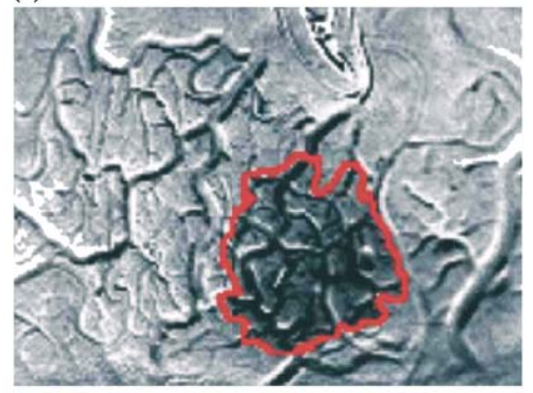

(c)

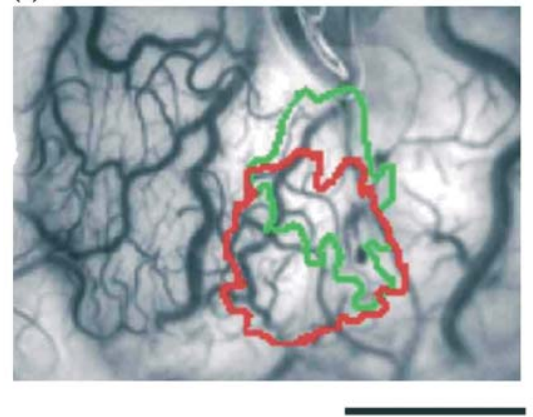

(d)

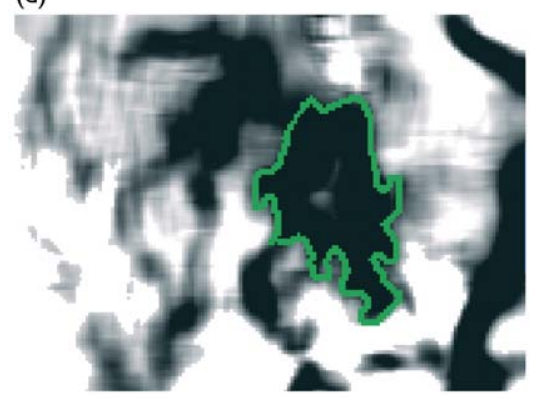

(e)
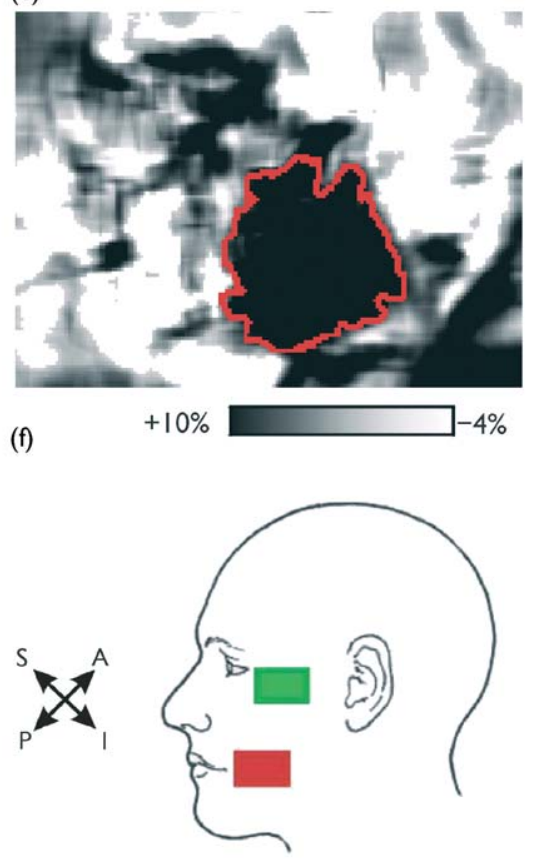

Fig. 3. Topography of face representation in SI. (a) Peri-orbital stimulation. (b) Peri-buccal stimulation. (c) Activation outlines on blood vessel map. $(\mathbf{d}, \mathbf{e})$ Low pass filtered images $(4 \times 4$ pixel gaussian moving window, $15 \%$ clip) used for determining activation outlines. (f) Location of stimulating electrodes. Regions with clearly separable blood vessel artifact were excluded. Bar $=5 \mathrm{~mm}$.

depth of field, was focused $300 \mu \mathrm{m}$ below the cortical surface [7].

The patient was maintained under general anesthesia (isoflurane $0.6 \%$ ) and the operating room lights were turned off during imaging. Images (field of view $14 \times 8.7 \mathrm{~mm}$, binned to $324 \times 240$ pixels) were acquired without synchronization to either heartbeat or respiration. We used a broadband filter $(650 \pm 40 \mathrm{~nm})$ to capture reflectance changes from a wider spectrum of sources, including light scatter, in order to increase the signal to noise ratio and minimize the required number of trials. Three stimulus conditions were presented (upper face stimulation, lower face stimulation, and blank (no stimulation)) in pseudo-randomized order with a $10-15 \mathrm{~s}$ interstimulus interval. Stimuli were presented through ball electrodes ( $2 \mathrm{~s}$, four $1.5 \mathrm{~ms} 2.2 \mathrm{~mA}$ electrical pulses at $2 \mathrm{~Hz}$ ) placed below and lateral to the left eye and along the left lower cheek parallel to the lips. For each stimulus, we collected 10 consecutive $300 \mathrm{~ms}$ image frames after stimulus onset and these were stored for subsequent analysis. We collected five blocks of five trials per stimulus.
Image analysis: For each stimulus condition, trials were summed to maximize signal-to-noise ratio. Because the face representation was located near the central sulcus, blood vessel artifact was at times substantial. As a result, noise-contaminated trials were excluded from analyses if the spatial map of the blank image was uneven or if the signal timecourse was not relatively flat. Single condition activation maps were generated by dividing stimulated conditions by the blank condition. Dark pixels in single condition maps indicate a response to that stimulus condition greater than that of the blank condition image; gray pixels indicate a response not different from blank.

Quantifying the evoked ORIS: Following low-pass filtering (4 pixel rectangular kernel), we delineated the regions of strongest activation with a thresholding procedure (top 15\%, $25 \%$, and $50 \%$ of the gray pixel value distribution) [8]. We also measured the time course of reflectance outside (control) and within the areas of activation. Time courses 
were derived directly from the raw reflectance signals and were independent of spatial filtering. Greater cortical activity corresponds to a darkening of the cortical tissue and a greater negative deflection of the signal time course.

\section{RESULTS}

Topography: We recorded topographically specific, focal activation of the upper $v$ s lower face. As shown in Fig. 2a,b (raw blank-subtracted images), lower face stimulation produced activation lateral to upper face stimulation. The blank condition (no facial stimulation) produced no significant changes in reflectance of light (Fig. 2c).

Each of three locations (the upper face site (Fig. 2a,e, yellow box), the lower face site (Fig. 2b,f, red box), and an unactivated site (Fig. 2c,g, blue box)) demonstrated stimulus specificity. Sampled locations were chosen away from large vessels, so that vascular noise could be minimized (e.g. the upper face site). Stimulation of the upper face produced a large negative reflectance change at the upper face site (Fig. 2e, yellow line, $\sim 6 \%$ ) and an increase in reflectance at the lower face site (red line). The activity recorded at the upper face site was significantly different from the activity recorded at the lower face or at control sites (one way repeated measure ANOVA, $p<0.001$; post hoc Holm-Sidak method, $p<0.05$ ). Similarly (Fig. 2f), stimulation of the lower face produced a greater reflectance change at the lower face site (red line) when compared to upper face (yellow line) or to control sites (blue line; repeated measure ANOVA, $p<0.001$; post hoc $p<0.05)$. Reflectance changes during the blank stimulus condition were small at all three sites $(>2 \%)$ and not significant (Fig. $2 \mathrm{~g}, p=0.2$ ), as were those at the control site (blue line; $<2 \%$ ), suggesting that baseline reflectance levels are $<2 \%$ (compare blue lines in Fig. 2e-g).

The timecourse of the change in reflectance (Fig. 2e,f, averaged over 5 trials) was characteristic of the intrinsic signal [9], beginning within $1 \mathrm{~s}$ after stimulus onset and peaking at $\sim 2-3 \mathrm{~s}$. We did not collect reflectance beyond $3 \mathrm{~s}$ post-stimulus onset, as previously published reports indicate the signal declines after this point $[6,10]$. Thus, stimulation of either upper face or lower face produced reflectance changes consistent with the known magnitude and timecourse of the intrinsic signal. They were topographically specific and were not due merely to noise or random alterations in vasomotor tone.

Area of activation: The activations were focal and did not appear to spread to adjacent gyri. To quantify the areas of activation, we took measurements from the images following low pass filtering and thresholding procedures (see Materials and Methods). Different threshhold values (15\%, $25 \%$ and $50 \%$ thresholds levels, corresponding to $1.5 \%, 2.5 \%$ and $5.0 \%$ of reflectance change, respectively) produced fairly similar activation areas (within a factor of 2). For example (Fig. 3), a 15\% threshhold produced a lower face activation area of roughly $5 \mathrm{~mm}$ in diameter (Fig. 3b,e, red outline, $0.18 \mathrm{~cm}^{2}$ ) and an upper face activation area $3-5 \mathrm{~mm}$ in diameter (Fig. $3 \mathrm{a}, \mathrm{d}$, green outline, $0.13 \mathrm{~cm}^{2}$ ). The lower face activation at a $25 \%$ threshhold was $18 \%$ larger $\left(0.21 \mathrm{~cm}^{2}\right)$ and at a $50 \%$ threshhold was $29 \%$ larger $\left(0.23 \mathrm{~cm}^{2}\right)$. Thus, both upper and lower face stimulation produced discrete activations that were similar in size.
Cortical magnification: We estimated the cortical magnification factor (the amount of cortex devoted to representing a region of the sensory periphery) by measuring the distance between the electrode contacts on the face $(\sim 7 \mathrm{~cm})$ and the distance between the centers of the change in reflectance on the cortical surface $(\sim 2.5 \mathrm{~mm})$. This produced a CMF of $0.36 \mathrm{~mm} / \mathrm{cm}(2.5 \mathrm{~mm} / 7 \mathrm{~cm})$ for facial skin.

\section{DISCUSSION}

In the context of a neurosurgical procedure ORIS represents a relatively non-invasive method for obtaining high-resolution maps of cortical functional activation. Although recent studies have seen an improved spatial resolution with BOLD fMRI methods [11], the temporal and spatial resolution of ORIS are typically an order of magnitude better than conventional 1.5 T BOLD fMRI. The resolution of ORIS is based on its sensitivity to the initial decrease in oxygenation and intracellular/extracellular fluid shifts, both of which are more tightly linked spatially with neuronal activity than BOLD methods [10,12].

Previous reports of intraoperative ORIS have demonstrated the feasibility of the technique for mapping language and somatosensory cortex [3-6,10,13]. However, most studies report large activation areas $9-20 \mathrm{~cm}^{2}$ for individual digits and $25 \mathrm{~cm}^{2}$ for median nerve stimulation $[3,5,10,13]$, although one study in somatosensory cortex reports comparable activation sizes [6] (but see [4,14]). Activations obtained in non-human primates $[5,8,9]$ under well-controlled laboratory conditions are much smaller. In monkeys, peripheral activation of somatosensory cortex elicits focal activations $0.1-1.0 \%$ in amplitude with a diameter of $1-$ $3 \mathrm{~mm}$ for individual digits and up to $10 \mathrm{~mm}$ for median nerve stimulation $[5,8]$. The results of our study thus approximate the monkey data and demonstrate improved spatial resolution.

We attribute the ability to obtain focal activation in human somatosensory cortex to several factors. In our experiments, the glass footplate on the cortical surface and camera were all fixed rigidly to the skull minimizing motion artifact. Rather than the lens of the surgical microscope, we used a tandem lens arrangement [7], providing a narrow depth of field which was focused beneath the surface vasculature, thereby reducing vasomotor artifacts and possibly decreasing the more diffuse perfusion component of the signal [5,10,12]. Finally, the broadband filter $(650 \pm 40 \mathrm{~nm})$, intended to capture reflectance changes from a wider spectrum of sources, may have contributed to the large signal size $(\sim 5 \%)$.

Another difference between our study and previous human studies $[5,6]$ was the source of stimulation. In contrast to nerve stimulation or mechanical vibrational stimulation, we used electrical stimulation of the skin (as did [6]), which, in monkey, produces similar activations as cutaneous stimulation (light tapping) $[8,15,16]$ and avoids possible contamination from motor cortical activation $[3,4,10,13]$.

Topography: Consistent with both electrical and imaging studies in humans $[6,17,18]$, we demonstrate in this human subject that peri-orbital skin is represented medial to lateral facial skin in Brodmann area 1 on the crest of the postcentral gyrus. These data are also consistent with topographical maps obtained from single unit recordings in Brodmann 
Area 1 of macaque and New World monkeys [19-21]. Significant overlap $(\sim 30 \%)$ was also observed between upper vs lower face activations (Fig. 3d). Such overlap is commonly described in the somatosensory literature $[5,8,16]$ and is attributed to the presence of large receptive fields which span multiple body parts and large receptive field scatter within single cortical sites.

Cortical magnification: Although somatosensory cortical magnification factors (CMF, $\mathrm{mm}$ cortex $/ \mathrm{mm}$ skin) in non-human primates have been studied extensively $[19,20,22-23]$, few data exist on somatosensory CMFs in the human, especially at the sub-millimeter scale. We estimated a CMF of $0.36 \mathrm{~mm} / \mathrm{cm}$ of facial skin. A comparable measurement from the face area of the cynomologus macaque is $0.5-1.0 \mathrm{~mm}$ cortex $/ \mathrm{cm}$ facial skin. In Figure 11 of [19], the distance between the center of cortical representation of orbital skin in Area 1 and penetrations 6-10 is 2-4 mm; and in Figures 13 and 19 from [21], the distance from upper face to lateral face is about $3 \mathrm{~mm}$. In the average $7-8 \mathrm{~kg}$ macaque, the distance between orbital skin and upper lip is about $4 \mathrm{~cm}$. In New World monkeys, similar Area 1 measurements give a CMF of about $1.0 \mathrm{~mm}$ cortex/ $\mathrm{cm}$ facial skin. In Figure 2 of [24] the distance between upper lip to lateral face in the squirrel monkey is $2 \mathrm{~mm}$. In Figure 3 and 4 of [25] the distance from upper lip and side of face is about $2 \mathrm{~mm}$. The distance from lateral orbit to upper lip in the squirrel monkey is about $2 \mathrm{~cm}$. Thus, our calculations suggest that the facial CMF in the human is smaller than in the macaque by a factor of $2-3$. However, given the degree of normal inter-individual somatotopic variability [22], the error in estimating from published reports, and other possible methodological considerations, we conclude that the human facial CMF is comparable with estimates from other primate species.

\section{CONCLUSION}

This report presents data from a single individual, where imaging quality was sufficiently high to make a significant contribution to the human imaging literature. We demonstrate in this human subject that peri-orbital skin is represented medial to lateral facial skin in Brodmann area 1 on the crest of the postcentral gyrus. We also find that the cortical magnification factor (cortical/cutaneous) for human face representation is on the order of $1 \times 10^{-2}$ and is comparable between human and primates.

\section{REFERENCES}

1. Bonhoeffer T and Grinvald A. Optical imaging based on intrinsic signals. The methodology. In: Togaand AW and Mazziota JC (eds). Brain Mapping. The Methods. San Diego: Academic Press; 1966, pp. 55-99.

2. Haglund MM, Ojemann GA and Hochman DW. Optical imaging of epileptiform and functional activity in human cerebral cortex. Nature 1992; 358:668-671.

3. Toga AW, Cannestra AF and Black KW. The temporal/spatial evolution of optical signals in human cortex. Cerebr Cortex 1995; 5:561-565.

4. Cannestra AF, Bookheimer SY, Pouratian N, O'Farrell A, Sicotte N, Martin NA et al. Temporal and topographical characterization of langauge cortices utilizing intraoperative optical intrinsic signals Neuroimage 2000;12:41-54.

5. Shoham D and Grinvald A. The cortical representation of the hand in macaque and human area S-1: high resolution optical imaging. J Neurosci $2001 ; 21: 6820-6835$.

6. Sato K, Nariai T, Sasaki S, Yazawa I, Mochida H, Miyakawa N et al Intraoperative intrinsic signal imaging of neuronal activity from subdivisions of the human primary somatosensory cortex. Cerebr Cortex 2002; 12:269-280.

7. Ratzlaff EH and Grinvald A. A tandem-lens epifluourescence macroscope: hundred-fold brightness advantage for wide field imaging. J Neurosci Methods 1991; 36:127-137.

8. Chen LM, Friedman RM, Ramsden BM, LaMotte RH and Roe AW. Fine-scale organization of S1 (Area 3b) in the squirrel monkey revealed with intrinsic optical imaging. J Neurophysiol 2001; 86:3011-3029.

9. Tommerdahl M, Delemos KA, Favorov OV, Metz CB, Vierck CJ and Whitsel BL. Response of anterior parietal cortex to different modes of same-site skin stimulation. J Neurophysiol 1998; 80:3272-3283.

10. Cannestra AF, Pouratian N, Bookheimer SY, Martin NA, Becker DP et al. Temporal spatial differences observed by functional MRI and human intraoperative optical imaging. Cerebr Cortex 2001; 11:773-782.

11. Duong TQ, Kim D-S, Ugurbil K and Kim S-G. Localized cerebral blood flow response at submillimeter columnar resolution. Proc Natl Acad Sci USA 2001; 98:10904-10909.

12. Vanzetta I and Grinvald A. Increased cortical oxidative metabolism due to sensory stimulation: implications for functional brain imaging. Science 1999; 286:1555-1558.

13. Cannestra AF, Black KL, Martin NA, Cloughesy T, Burton JS, Rubinstein E et al. Topographical and temporal specificity of human intraoperative optical intrinsic signals. Neuroreport 1998; 9:2557-2563.

14. Pouratian N, Sicotte N, Rex D, Martin NA, Becker D, Cannestra AF et al. Spatial/temporal correlation of BOLD and optical intrinsic signals in humans. Magn Reson Med 2002; 47:766-776.

15. Pollok B, Moll M, Schmitz F, Muller K and Schnitzler A. Rapid mapping of finger representations in human primary somatosensory cortex applying neuromagnetic steady-state responses. Neuroreport 2002; 13:235-238.

16. Schroeder CE, Seto S, Arezzo JC and Garraghty PE. Electrophysiological evidence for overlapping dominant and latent inputs to somatosensory cortex in squirrel monkeys. J Neurophysiol 1995; 74: 722-732.

17. Penfield W and Rasmussen T. The Cerebral Cortex of Man: A Clinical Study of Localization of Function. New York: MacMillan; 1950.

18. ematsu S, Lesser R, Fisher RS, Gordon B, Hara K et al. Motor and sensory cortex in human: topography studied with chronic subdural stimulation. Neurosurgery 1992; 31:59-72.

19. Nelson RJ, Sur M, Felleman DJ and Kaas JH. Represenation of the body surface in postcentral parietal cortex of Macaca fascicularis. J Comp Neurol 1980; 192:611-643.

20. Sur M, Nelson RJ and Kaas JH. Representations of the body surface in cortical areas $3 \mathrm{~b}$ and 1 of squirrel monkeys: comparisons with other primates. J Comp Neurol 1982; 211:177-192.

21. Pons TP, Garraghty PE, Cusick CG and Kaas JH. A sequential representation of the occiput, arm, forearm and hand across the rostrocaudal dimension of areas 1,2 and 5 in macaque monkeys. Brain Res 1985; 335:350-353.

22. Sur M, Merzenich MM and Kaas JH. Magnification, receptive-field area, and "hypercolumn" size in areas $3 \mathrm{~b}$ and 1 of somatosensory cortex in owl monkeys. J Neurophysiol 1980; 44:295-311.

23. Jain N, Qi H-X, Catania KC and Kaas JH. Anatomic correlates of the face and oral cavity representations in the somatosensory cortical area $3 \mathrm{~b}$ of monkeys. J Comp Neurol 2001; 429:455-468.

24. Cusick CG, Wall JT and Kaas JH. Representations of the face, teeth and oral cavity in areas $3 \mathrm{~b}$ and 1 of somatosensory cortex in squirrel monkeys. Brain Res 1986; 370:359-364.

25. Manger PR, Woods TM and Jones EG. Representation of the face and intraoral structures in area $3 \mathrm{~b}$ of the squirrel monkey (Saimiri sciureus) somatosensory cortex, with special reference to the ipsilateral representation. J Comp Neurol 1995; 362:597-607. 